\title{
An Intelligence Approach for Porosity and Permeability Prediction of Oil Reservoirs using Seismic Data
}

\author{
Edris Joonaki \\ Ahwaz Faculty of Petroleum \\ Petroleum University of \\ Technology, Ahwaz, Iran
}

\author{
Shima Ghanaatian \\ Ahwaz Faculty of Petroleum \\ Petroleum University of \\ Technology, Ahwaz, Iran
}

\author{
Ghassem Zargar \\ Ahwaz Faculty of Petroleum \\ Petroleum University of \\ Technology, Ahwaz, Iran
}

\begin{abstract}
Nowadays the main challenge is to obtain a method for the estimation of key reservoir parameters with the lowest possible estimation error. Accurate reservoir characterization requires the integration of core and log data to understand the variation in hydraulic properties such as porosity, permeability and capillary pressure. Time-lapse seismic can be used as an important tool in reservoir characterization, monitoring and management. Reservoir parameters are converted to seismic parameters by using the rock physics models. This paper presents an analysis and explanation of an approach of developing rock physics model, and explains how the input data can be obtained to the model. And also this study presents an intelligence approach for the oil reservoir characterization by using seismic elastic properties and rock physics model together with minimum estimation error.
\end{abstract}

\section{General Terms}

Artificial Intelligence, Genetic Algorithm, Reservoir Characterization

\section{Keywords}

Artificial intelligence, Geophysics, Porosity, Permeability, Norne oil field, Reservoir characterizations

\section{INTRODUCTION}

In time-lapse or "4D" seismic projects, the objective is to infer fluid production from two or more seismic surveys recorded at different times in the reservoirs production life cycle. Rock physics modeling allows us to make these "time shifts" by changing saturation, pore pressure, and even porosity in the key reservoir intervals. Estimation of permeability and porosity using seismic data is a new challenge in the oil industry. Porosity and permeability are two of the most important parameters in most reservoir simulation models, and they have a large impact on reserve estimates, production forecasts and the economical evaluation of the reservoirs.

The process of oil or gas production causes variations in reservoir parameters such as fluid types, fluid saturation, pressure, temperature and viscosity, and thus changes seismic properties of saturated reservoir rock [1]. Converting saturations and pressure changes into seismic properties such as $\mathrm{P}$-wave velocity, S-wave velocity and density changes requires information about the rock properties. The dependence between fluid saturation changes and the seismic parameters are described by rock physics models (RPMs).
Once these rock physics relationships are established for a given reservoir rock, the seismic forward modeling can be done. That means converting a given pressure and fluid saturation state for a given reservoir rock into a seismic section. Moreover, this procedure acts as a bridge that relates seismic parameter changes to reservoir parameter changes and vice versa. This means that rock physics is a key element in such a process. Several theories and empirical relations link seismic properties of reservoir rock to pore spaces, pore fluids, effective pressure and other reservoir parameters. For the developing of RPMs, elastic moduli and densities of fluid, grain and dry rock are input data and they can be computed using empirical or theoretical relations. Even relations from laboratory measurements of core samples are important in developing of RPMs, empirical relations work only for specific situations and theoretical models have limitations due to their assumptions [2]. This paper makes an analysis on method of constructing Duffy-Mindlin's model for saturated reservoir rock and gives a brief introduction to the model.

This work aims at improving previous studies and presenting a robust, general and mathematically sound methodology for reservoir characterization. Our approach contains two new elements compared to the previous works:

- The use of rock physics models,

- The use of artificial intelligence for estimations.

In this paper we present an intelligence method for a joint estimation of porosity and permeability, which is generated by using field data from an oil field in Norway and using the rock physics models.

\section{ROCK PHYSIC MODELS}

A saturated porous reservoir rock contains of rock matrix and fluid, which can be considered as solid and fluid phases, respectively; when the pore has no fluid, the porous rock can be named as dry rock. Oil production from a reservoir makes alterations in fluid phase, while almost no changes in solid phase. Seismic wave velocities in a porous medium saturated with water depend on three constants, namely the bulk modulus $(\mathrm{K})$, shear modulus $(\mu)$ and density $(\rho)$. The bulk modulus or incompressibility of an isotropic rock is explained as the ratio of hydrostatic stress to volumetric strain. In other words, it knows us how difficult it is to compress the rock. The shear modulus or shear stiffness of the rock is explained as the ratio of shear stress to shear strain and in other words, how difficult it is to alter the shape of a rock sample. Han and 
Batzle (2004) have indicated that the bulk modulus of dry and water-saturated rocks, which are symbolized as $K_{d}$ and $K_{w}$, respectively, are more sensitive to the saturation of water than the P-wave velocity $\left(\mathrm{V}_{\mathrm{p}}\right)$ under the same condition of pressure, further, the alteration in water saturation has little influences on the shear modulus since the shear modulus of fluid $\left(\mu_{\mathrm{f}}\right)$ is zero. Since bulk modulus $\mathrm{K}$ is the most sensitive to the fluid saturation [2], Gassmann's model [3] calculates $V_{p}$ and $\mathrm{V}_{\mathrm{s}}$ of saturated rock by calculating $\mathrm{K}$ together with $\mu$ and $\rho$ for saturated reservoir rocks, which are $K_{s}, \mu_{s}$ and $\rho_{s}$, respectively, in order to effectively reflect effects of fluid substitution. Duffy-Mindlin's model [4] believes $V_{P}$ and $V_{S}$ as functions of not only $\rho_{\mathrm{s}}, \mathrm{K}_{\mathrm{f}}, \mathrm{Kgr}, \mu_{\mathrm{f}}$, and $\mu_{\mathrm{gr}}$ but also Young modulus $(E)$ and Poison ratio $(\gamma)$. However, since $E$ and $\gamma$ are explained as functions of $K_{d}$ and $\mu_{d}$, all parameters that are necessary for the construction of RPM in Duffy-Mindlin model are the same with Gassmann's equation. Duffy and Mindlin [4][5] assumed array of identical spheres as a facecentered cubic to calculate Young modulus $(E)$ and Poison ratio $(\gamma)$ as:

$$
\begin{aligned}
& E=\frac{9 K_{d} \mu_{d}}{3 K_{d}+\mu_{d}} \\
& \gamma=\frac{3 K_{d}-2 \mu_{d}}{2\left(3 K_{d}+\mu_{d}\right)}
\end{aligned}
$$

Where $K_{d}$ is bulk modulus of dry rock, and $\mu_{d}$ is shear modulus of dry rock. Derives seismic velocities as a function of $E$ and $\gamma$, effective pressure $\left(\mathrm{P}_{\mathrm{e}}\right)$ and base parameters as:

$V_{p}{ }^{2}=\frac{\left[C_{11}+\frac{\left(1-\frac{C_{11}+2 C_{12}}{3 K_{s}}\right)}{\frac{\phi}{K_{f}}+\frac{1-\phi}{K_{s}}+\frac{C_{11}+2 C_{12}}{3 K_{s}{ }^{2}}}\right]}{\rho_{d}}$

$V_{s}^{2}=\frac{C_{11}+C_{12}}{2 \rho_{d}}$

Where $K_{s}$ is bulk modulus of saturated rock, $\rho_{d}$ is density of dry rock, $\phi$ is the effective porosity of the medium, $\mathrm{K}_{\mathrm{f}}$ is bulk modulus of pore fluid that is calculated by using Wood's relation [6]:

$$
\frac{1}{K_{f}}=\frac{S_{o}}{K_{o}}+\frac{S_{g}}{K_{g}}+\frac{S_{w}}{K_{w}}
$$

Where $\mathrm{K}_{\mathrm{v}}, \mathrm{K}_{\mathrm{g}}$ and $\mathrm{K}_{\mathrm{w}}$ are bulk modulus of oil, gas and water respectively. And $\mathrm{C}_{11}$ and $\mathrm{C}_{12}$ are obtained, respectively, as:

$$
\begin{aligned}
& C_{11}=\frac{4-3 \gamma}{2-\gamma}\left\langle\frac{3 E^{2} P_{e}}{8\left(1-\gamma^{2}\right)^{2}}\right\rangle^{\frac{1}{3}} \\
& C_{12}=\frac{\gamma}{2(2-\gamma)}\left\langle\frac{3 E^{2} P_{e}}{8\left(1-\gamma^{2}\right)^{2}}\right\rangle^{\frac{1}{3}}
\end{aligned}
$$

With

$$
P_{e}=P_{\text {overburden }}-P_{\text {pore }}
$$

The bulk modulus of saturated rock can be calculated by using Gassmann's equation. It can be shown as the following equation [7]:

$$
K_{s}=K_{d}+\frac{\left(1-\frac{K_{d}}{K_{f r}}\right)^{2}}{\frac{\phi}{K_{f}}+\frac{1-\phi}{K_{f r}}+\frac{K_{d}}{K_{f r}{ }^{2}}}
$$

Where $\mathrm{K}_{\mathrm{fr}}$ is the bulk modulus of the solid frame work, $\phi$ is the effective porosity of the medium, $\mathrm{K}_{\mathrm{f}}$ is bulk modulus of pore fluid that is determined by using Wood's relation. DuffyMindlin's model consists of most important effects such as porosity, lithology, and mechanical compaction and also for fluid because of presence of fluid bulk modulus, $\mathrm{K}_{\mathrm{f}}$. Furthermore, the model considers the presence and variations of effective pressure, $\mathrm{P}_{\mathrm{e}}$. Hence, in the presence of shale compaction, the model must be employed even though the model is more complex than Gassmann's equation. The advantages \& disadvantages of Duffy-Mindlin's model are presented in Table1.

There are two ways of empirical relations and theoretical models for calculating $\mu_{\mathrm{d}}$ and $\mathrm{K}_{\mathrm{d}}$. empirical relation can be best if we can obtain the values of $K_{d}$ and $\mu_{d}$ from laboratory measurements of core samples. However, since core measurements are not always valid, we often calculate $K_{d}$ and $\mu_{\mathrm{d}}$ by using empirical relations such as Geertsma and Smith [8]. Here is the empirical relation that investigates the relation of dry moduli, grain moduli, porosity:

$$
\frac{1}{A_{d}}=\frac{1}{A_{f r}}(1+50 \phi)
$$

Where $A_{d}$ is either $K_{d}$ or $\mu_{d}$ and $\phi$ is porosity.

Theoretical models which compute the effective elastic moduli based on assumptions of contact surfaces between grains such as Hertz-Mindlin [9]. The Hertz-Mindlin model is employed to explain seismic parameter alterations due to pressure changes. The model presents [10]:

$$
\begin{aligned}
& K_{d}=\sqrt[n]{\frac{C^{2}(1-\phi)^{2} \mu^{2} P_{e}}{18 \pi^{2}(1-\gamma)^{2}}} \\
& \mu_{d}=\frac{5-4 \gamma}{5(2-\gamma)}\left(\sqrt[n]{\frac{3 C^{2}(1-\phi)^{2} \mu^{2} P_{e}}{2 \pi^{2}(1-\gamma)^{2}}}\right)
\end{aligned}
$$

Where $\mathrm{P}_{\mathrm{e}}$ is the effective pressure, $\mu$ is the shear modulus of the solid phase, $\gamma$ is poisson's ratio and $\mathrm{n}$ is the coordination number. In the original Hertz-Mindlin theory $\mathrm{n}$ is identical to 3. Some laboratory measurements of samples proposed a larger number for $\mathrm{n}$. Vidal [11] discovered $\mathrm{n}=5.6$ for $\mathrm{P}$ waves and $\mathrm{n}=3.8$ for shear waves for gas sands, while Landrø [12] employed $n=5$ for oil sands. We use $n=5$ in this study. $\mathrm{C}$ is the average number of contact points between the grains in a volume fraction, related with sorting, shape and packing of the grains, and porosity [13]. It is explained as:

$C=20-34 \phi+14 \phi^{2}$

The effective pressure employed in Hertz-Mindlin theory is considered as the difference between the lithostatic $\mathrm{P}_{\mathrm{ext}}$ and the hydrostatic pressure P [14]:

$$
P_{e}=P_{e x t}-\eta P
$$


Where $\eta$ is the coefficient of internal deformation that is commonly an unknown parameter. Table 2 presents the seismic elastic parameters of Norne oil field for this study.

\section{CASE DESCRIPTION}

The field to be studied is an oil reservoir in the Norne field. It is located in the blocks $6608 / 10$ and $6508 / 10$ on a horst block in the southern part of the Nordland II area in the Norwegian Sea. The rocks within the Norne reservoir are of late Triassic to middle Jurassic age. It was discovered in 1991, and oil production started in 1997. The Norne Field contains two separate oil compartments, the Norne Main Structure, Norne C-, D and E-segment, and the Northeast segment, Norne Gsegment. In this study we use the Norne E-segment data (Figure 1). The present geological model contains five reservoir zones. They are Garn, Not, Ile, Tofte and Tilje. Oil is principally found in the Ile and Tofte Formations, and gas in the Garn formation. The sandstones are buried at a depth of $2500-2700 \mathrm{~m}$. The porosity is in the range of $25-30 \%$, while permeability changes from 20 to $2500 \mathrm{mD}$ [15].

\section{PROPOSED METHODOLOGY}

\subsection{GENETIC ALGORITHM}

The first step in GA method, like any other optimization algorithms is clarifying the objective function and its decision variables. Basic components of genetic algorithm are gene, chromosome and population. Genetic algorithm operates on a finite set of chromosomes (points) which are named population. The different populations are explained as gens. Population size, mutation probability and crossover probability are the control parameters that are used in genetic algorithm. Genetic algorithms are generally executed as follows:

1- Explain the problem as an objective function that shows the fitness, $f_{i}$, of each candidate solution, $\alpha_{i}$, that is named chromosome.

2- Randomly initialize a population created by a certain number of chromosomes, with each chromosome representing a candidate solution to the problem.

3- Assign each chromosome a fitness score in accord with to the objective function. If the chromosome population satiates the convergence tolerance, it is selected as the solution to the optimization problem. If not, the following steps are taken.

4- Produce a mating pool of the chromosomes from the existing population.

5- Create offsprings from the chromosomes in the mating pool by means of a crossover operator.

6- Change some of the chromosomes by the mutation operator.

7- Go back to step 3 .

Figure 2 indicates general Genetic algorithm scheme. Genetic algorithm is applicable for optimizing the design parameters formed into a corporation into a specified fitness function to achieve a goal fitness quantity. The key characteristic of genetic algorithms and other similar algorithms is that they are derivative-free. In fact, the stochastic nature of the algorithm with dynamic evaluation of the fitness function changes it into a powerful systematic random search engine. This approach is an alternative to incapable derivative-based methods. This extends its ability to a wide range of applications. Recently this approach has been employed to many different optimization problems including non-linear geophysical inversion [16].

\subsection{MULTI AND SINGLE OBJECTIVE OPTIMIZATION}

In single objective optimization we take into account minimizing or maximizing only one objective, without considering the effect of other criteria. Multi-objective optimization is delineated as the task of finding one or more optimum solutions when a problem contains more than one objective or goal. Unlike single objective that handles a single space (decision variable space), in multi-objective optimization, a new space is also taken into account which is named the objective function space. In a multi-objective optimization problem, the decision vector is symbolized by $\mathrm{x}$ and the decision space is shown by X. Similarly, the objective vector is symbolized by $\mathrm{y}$ and $\mathrm{Y}$ represents the objective space. The multi-objective optimization, in general form, can be explained as:

$\begin{array}{ll}\text { Maximize/Minimize } f_{m}(x), & \mathrm{m}=1,2,3, \ldots, \mathrm{M} \\ \text { Subject to } \quad g_{l}(x) \geq 0 & 1=1,2,3, \ldots, \mathrm{L} \\ H_{t}=0 & \mathrm{t}=1,2,3, \ldots, \mathrm{T}\end{array}$

Where solution $\mathrm{x}$ is a decision vector of $\mathrm{n}$ variables, $\mathrm{x}=(\mathrm{x} 1$, $\mathrm{x} 2, \ldots, \mathrm{xn}) . \mathrm{M}$ is the number of objective functions in the problem which can be minimized or maximized: $f(x)=(f l(x)$, $f 2(x), \ldots, f M(x))$. The multi-objective optimization problem also may have constraint functions $(\mathrm{gl}(\mathrm{x})$ and $\mathrm{Ht}$ ) which compute the set of feasible solutions. In any single objective optimization, the optimal solution is the one that presents maximum (or minimum) value of the objective function. However, in the context of a multi-objective optimization, the notion of optimality is different and we are curious in finding good compromises among the objectives that we hope to optimize.

Although multi-objective optimization algorithms have been widely employed in some engineering problems, their applications to petroleum engineering problems are still limited. Traditionally the oil industry employs some form of weighted sum approach for handling multiple objectives without considering the limits of this approach. Dal Moro and Pipan [17] employed multi-objective evolutionary algorithm for the joint inversion of seismic surface wave dispersion curves and reflection travel times. Boomer and Brazier [18] also suggested a new method to achieve velocity models from inversion of seismic data based on a non-dominated sorting genetic algorithm.

In this paper we use the multi-objective optimization and Genetic Algorithm for reservoir characterization with minimum errors by using seismic elastic properties.

\section{RESULTS AND DISCUSSIONS}

Two different rock physic models have been modified to determine bulk modulus of dry rock, namely Geertsma and Hertz-Mindlin models. The models have been modified by defining a set of coefficients to be adjusted by Genetic Algorithm (GA) optimization.

Multi-objective GA has been used to estimate the optimal coefficients so that $\mathrm{V}_{\mathrm{p}}, \mathrm{V}_{\mathrm{s}}$ and $\rho_{\mathrm{s}}$ are estimated by the 
Geertsma and Hertz-Mindlin models as close to measurements as possible. Measurements are presented in Table 2. Estimated values are shown in Table 3 and associated errors in Table 4. According to the results, the modified Hertz-Mindlin model is a considerably more accurate predictor, compared with the modified Geertsma model. For each run of Genetic Algorithm, Porosity and permeability of each formation were calculated using the Duffy-Mindlin's model and Hertz-Mindlin model, respectively presented in Tables 5 and 6 . Corresponding horizontal permeability realizations were created by empirical porosity permeability relations that are presented in Table 7, and Table 8 illustrates the current reservoir zonation which is used in the simulation model [19].

\section{CONCLUSION}

Rock physics models explain crucial relations between reservoir parameters and seismic properties of reservoir rock and they are very important not only for a time-lapse seismic project but also for the reservoir characterization of one reservoir. In this work it was proved that the Duffy-Mindlin's model works properly at porous reservoir, especially in case of presence of shale compaction In the case, we need to use not only empirical relations for constructing rock physics model but also a contact theoretical model for the calculation of dry moduli. Geertsma's empirical relation is often used due to its consistency to Duffy-Mindlin's model. Among theoretical models, Hertz-Mindlin's theoretical model is the most popular one, since the other theoretical models are based on the model of Hertz- Mindlin. This paper has shown the modified Hertz-Mindlin model is a considerably more accurate predictor, compared with the modified Geertsma model, and it is more suitable for calculation of dry moduli. Also it is proved that Genetic Algorithms are a feasible technique for generating reservoir characterization using timelapse seismic data. The method is capable of handling many parameters, which is critical when dealing with large full-field reservoir simulation models. This paper has proved the application of a Genetic Algorithm to a realistic case, with respect to main issues of model's formulations for the reservoir characterizations.

\section{ACKNOWLEDGMENT}

The authors want to thank the Norwegian University of Science and Technology (NTNU), the StatoilHydro (operator of the Norne Field), for the release and availability of the Norne Field data on the Internet.

\section{NOMENCLATURE}

$\mathrm{K}$ : Bulk modulus

$\mathrm{K}_{\mathrm{d}}$ : Bulk modulus of dry rock

$\mathrm{K}_{\mathrm{w}}$ : Bulk modulus of water-saturated rock

$\mathrm{K}_{\mathrm{s}}$ : Bulk modulus of saturated rock

$\mathrm{K}_{\mathrm{fr}}$ : Bulk modulus of the solid framework

$\mathrm{K}_{\mathrm{f}}$ : Bulk modulus of pore fluid

$\mathrm{K}_{\mathrm{o}}$ : Bulk modulus of oil

$\mathrm{K}_{\mathrm{g}}$ : Bulk modulus of gas

$\mathrm{K}_{\mathrm{w}}$ : Bulk modulus of water

$\mathrm{V}_{\mathrm{p}}$ : P-wave velocity

$\mathrm{V}_{\mathrm{s}}$ : S-wave velocity $\mu$ : Shear modulus

$\mu_{\mathrm{f}}$ : Shear modulus of pore fluid

$\mu_{\mathrm{s}}$ : Shear modulus of saturated rock

$\mu_{\mathrm{d}}$ : Shear modulus of dry rock

$\rho$ : Density

$\rho_{\mathrm{d}}$ : Density of dry rock

$\mathrm{S}_{\mathrm{w}}:$ Water saturation

$\mathrm{S}_{\mathrm{g}}$ : Gas saturation

$P_{\mathrm{e}}$ : Effective pressure

$\mathrm{S}_{\mathrm{o}}$ : Oil saturation

$\phi$ : Porosity

$\gamma$ : Poisson's ratio

$\mathrm{P}_{\mathrm{ext}}$ : Lithostatic pressure

P: Hydrostatic pressure

$E$ : Young modulus

Table 1. Advantages \& Disadvantages for Duffy-Mindlin's model

\begin{tabular}{|c|c|}
\hline $\begin{array}{c}\text { Advantages } \\
\text { especially in the case of presence } \\
\text { of shale compaction }\end{array}$ & Disadvantages \\
\hline $\begin{array}{c}\text { Fairly complex } \\
\text { calculations }\end{array}$ \\
\hline $\begin{array}{c}\text { Take into account almost effects } \\
\text { seismic responses } \\
\text { servoir parameters on }\end{array}$ & $\begin{array}{c}\text { Essentially need to } \\
\text { obtain dry modulus }\end{array}$ \\
\hline $\begin{array}{c}\text { The estimation of the effects of } \\
\text { fluid substitution would be well } \\
\text { to make comparison between } \\
\text { production stages }\end{array}$ & ------------- \\
\hline
\end{tabular}


Table 2. Seismic elastic parameters from Norne oil field [20]

\begin{tabular}{|c|c|c|}
\hline Overburden & $\begin{array}{l}V_{p} \\
V_{s} \\
\rho_{s}\end{array}$ & $\begin{array}{l}3350 \\
1800 \\
2450\end{array}$ \\
\hline Inside reservoir & $\begin{array}{l}V_{p} \\
V_{s} \\
\rho_{s}\end{array}$ & $\begin{array}{l}3200 \\
1600 \\
2300\end{array}$ \\
\hline Under reservoir & $\begin{array}{l}\mathrm{V}_{\mathrm{p}} \\
\mathrm{V}_{\mathrm{s}} \\
\rho_{\mathrm{s}}\end{array}$ & $\begin{array}{l}3500 \\
1900 \\
2450\end{array}$ \\
\hline Garn formation & $\begin{array}{c}\mathrm{K}_{\mathrm{fr}} \\
\mu_{\mathrm{fr}} \\
\mathrm{K}_{\mathrm{ma}} \\
\rho_{\mathrm{ma}}\end{array}$ & $\begin{array}{c}19-37 \phi \\
12-21.5 \phi \\
37 \mathrm{GPa} \\
2650 \mathrm{~kg} / \mathrm{m}^{3}\end{array}$ \\
\hline $\begin{array}{l}\text { Ile, Tilje, Tofte } \\
\text { formations }\end{array}$ & $\begin{array}{c}\mathrm{K}_{\mathrm{fr}} \\
\mu_{\mathrm{fr}} \\
\mathrm{K}_{\mathrm{ma}} \\
\rho_{\mathrm{ma}}\end{array}$ & $\begin{array}{c}18.5-27.5 \phi \\
11-13 \phi \\
37 \mathrm{GPa} \\
2650 \mathrm{~kg} / \mathrm{m}^{3}\end{array}$ \\
\hline $\begin{array}{l}\text { Bulk modulus of oil } \\
\text { Bulk modulus of gas } \\
\text { Bulk modulus of water } \\
\text { Water density } \\
\text { Gas density } \\
\text { Oil density } \\
\text { Salinity }\end{array}$ & $\begin{array}{c}K_{o} \\
K_{g} \\
K_{w} \\
\rho_{w} \\
\rho_{g} \\
\rho_{o} \\
S A L\end{array}$ & $\begin{array}{c}1 \mathrm{GPa} \\
0.1 \mathrm{GPa} \\
2.7 \mathrm{GPa} \\
1000 \mathrm{~kg} / \mathrm{m}^{3} \\
190 \mathrm{~kg} / \mathrm{m}^{3} \\
860 \mathrm{~kg} / \mathrm{m}^{3} \\
0.05 \mathrm{ppm}\end{array}$ \\
\hline
\end{tabular}

Table 3. Estimated values of $V_{p}, V_{s}$ and $\rho_{s}$ by the modified models

\begin{tabular}{|c|c|c|c|c|c|c|c|}
\hline Geertsma Model & $\mathrm{V}_{\mathrm{P}}$ & $\mathrm{V}_{\mathrm{s}}$ & $\rho_{\mathrm{s}}$ & Hertz-Mindlin Model & $\mathrm{V}_{\mathrm{p}}$ & $\mathrm{V}_{\mathrm{s}}$ & $\rho_{\mathrm{s}}$ \\
\hline Overburden & 2713 & 2646 & 1813 & Overburden & 3149 & 1926 & 2254 \\
\hline Inside reservoir & 2528 & 2416 & 1794 & Inside reservoir & 3072 & 1696 & 2093 \\
\hline Under reservoir & 2905 & 2831 & 1862 & Under reservoir & 3290 & 1995 & 2327 \\
\hline
\end{tabular}

Table 4. Estimation errors of the modified models in percent

\begin{tabular}{|c|c|c|c|c|c|c|c|}
\hline Geertsma Model & $\mathrm{V}_{\mathrm{P}}$ & $\mathrm{V}_{\mathrm{s}}$ & $\rho_{\mathrm{s}}$ & Hertz-Mindlin Model & $\mathrm{V}_{\mathrm{p}}$ & $\mathrm{V}_{\mathrm{s}}$ & $\rho_{\mathrm{s}}$ \\
\hline Overburden & 19 & 47 & 26 & Overburden & 6 & 7 & 8 \\
\hline Inside reservoir & 21 & 51 & 22 & Inside reservoir & 4 & 6 & 9 \\
\hline Under reservoir & 17 & 49 & 24 & Under reservoir & 6 & 5 & 5 \\
\hline
\end{tabular}


Table 5. The calculated porosity distribution of formations

\begin{tabular}{|c|c|}
\hline Formations & Porosity \\
\hline Garn formation & $0.23 \leq \phi \leq 0.34$ \\
\hline Ile and Tofte formation & $0.19 \leq \phi \leq 0.31$ \\
\hline Tilje formation & $0.18 \leq \phi \leq 0.29$ \\
\hline
\end{tabular}

Table 7. Porosity-Permeability relations for each layer

\begin{tabular}{|c|c|}
\hline Layer number & Relation \\
\hline 1 & $\log _{10}(\mathrm{~K})=7.2 \phi+0.6$ \\
\hline 2 & $\log _{10}(\mathrm{~K})=31.4 \phi-3$ \\
\hline 3 & $\log _{10}(K)=23.5 \phi-4$ \\
\hline 5,6 & $\log _{10}(K)=16.8 \phi-2$ \\
\hline 7,8 & $\log _{10}(K)=18.2 \phi-2.5$ \\
\hline 9 & $\log _{10}(\mathrm{~K})=22.5 \phi-3.3$ \\
\hline 10 & $\log _{10}(K)=13.96 \phi-0.85$ \\
\hline 11 & $\log _{10}(K)=17.1 \phi-2.1$ \\
\hline 12 & $\log _{10}(K)=17.3 \phi-2.11$ \\
\hline 13 & $\log _{10}(K)=13.3 \phi-0.9$ \\
\hline 14 & $\log _{10}(K)=20.32 \phi+3$ \\
\hline 15 & $\log _{10}(K)=15.83 \phi-1.9$ \\
\hline 16 & $\log _{10}(\mathrm{~K})=12.7 \phi-0.24$ \\
\hline 17 & $\log _{10}(K)=19 \phi-1.95$ \\
\hline 18 & $\log _{10}(\mathrm{~K})=14.1 \phi-0.87$ \\
\hline 19 & $\log _{10}(K)=21.7 \phi-2.07$ \\
\hline 20 & $\log _{10}(K)=15.1 \phi-1.09$ \\
\hline 21,22 & $\log _{10}(K)=23.98 \phi-2.6$ \\
\hline
\end{tabular}

Table 6. The calculated permeability distribution of formations $(\mathrm{mD})$

\begin{tabular}{|c|c|}
\hline Formations & Permeability \\
\hline Garn formation & $36 \leq \mathrm{K} \leq 1243$ \\
\hline Ile and Tofte formation & $24 \leq \mathrm{K} \leq 1996$ \\
\hline Tilje formation & $51 \leq \mathrm{K} \leq 2231$ \\
\hline
\end{tabular}

Table 8. Reservoir zonation from the simulation model

\begin{tabular}{|c|c|}
\hline Layer number & Formation name \\
\hline $1,2,3$ & Garn \\
\hline $5,6,7,8,9,10,11$ & Ile \\
\hline $12,13,14,15,16,17,18$ & Tofte \\
\hline $19,20,21,22$ & Tilje \\
\hline
\end{tabular}

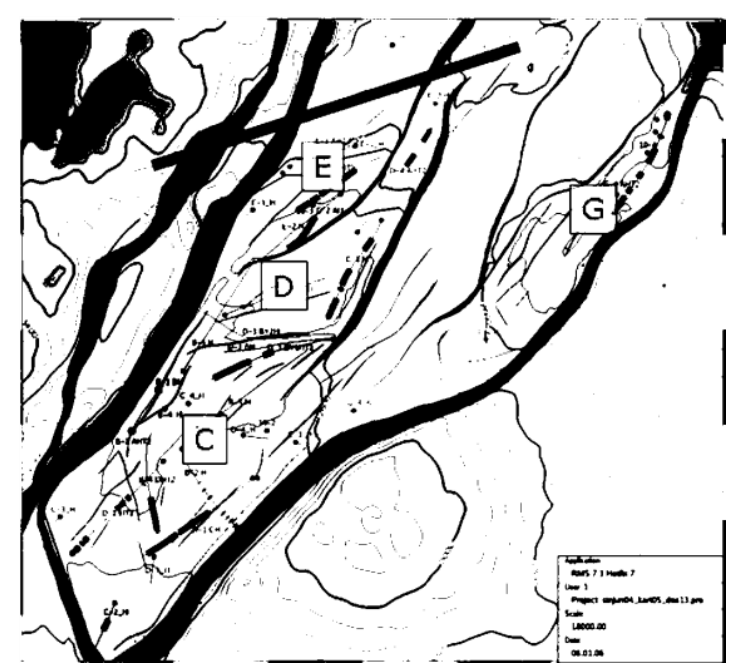

Figure 1. Top view of Norne field showing E-segment used in this study 


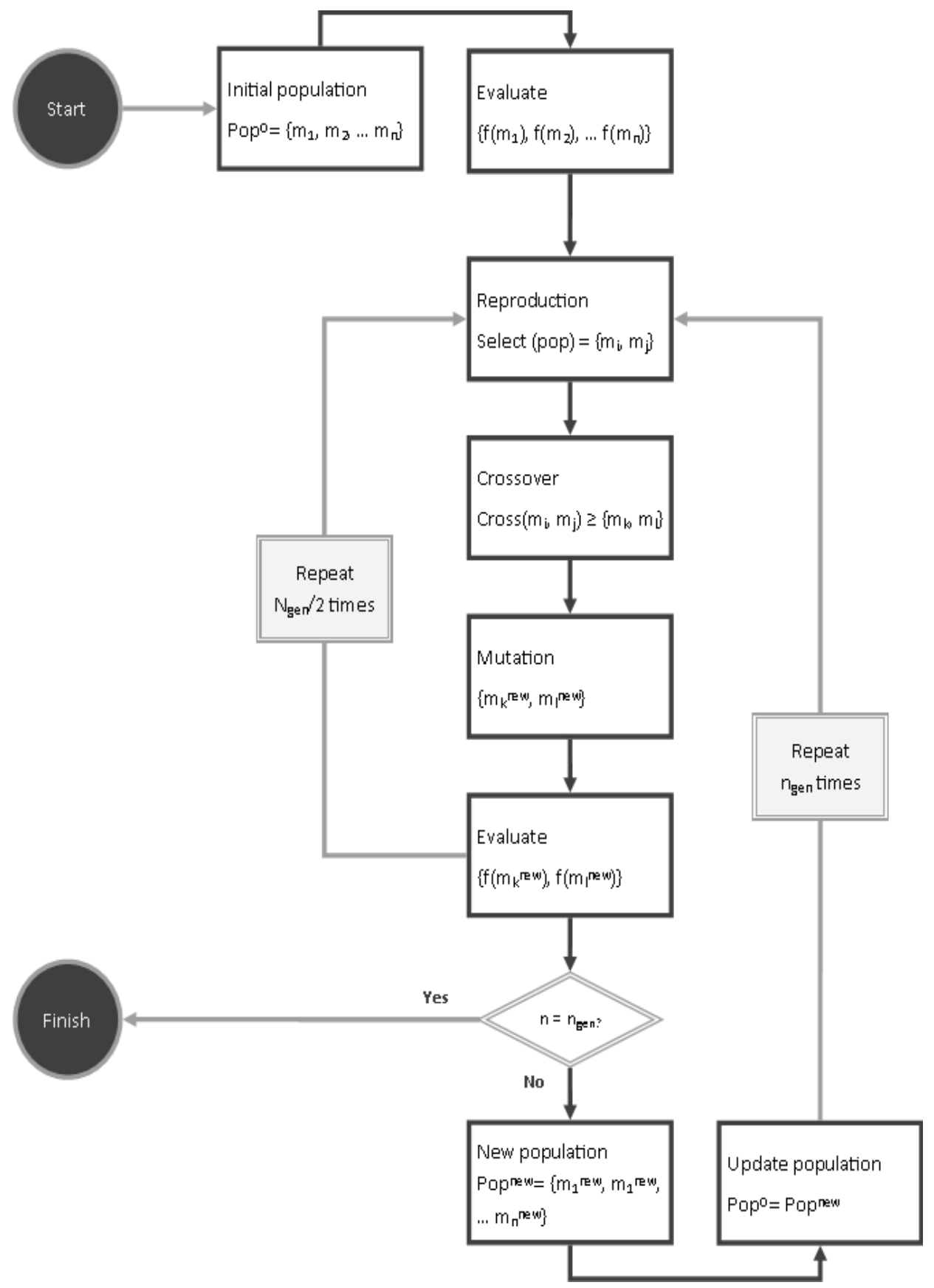

Figure 2. Schematic diagram of Genetic Algorithm

\section{REFERENCES}

[1] Lumley D E, Behrens R A and Wang Z, 1997, Assessing the technical risk of a $4 \mathrm{D}$ seismic project, The Leading Edge, 161287-1292.

[2] Han, D.-H.andBatzle, M.L., 2004, Gassmann's equation and fluid- saturation effects on seismic velocities, Geophysics, p. 398-405.

[3] Gassmann, F., 1951, U " ber die Elastizita " tporo " serMedien.Vier.derNatur.

Gesellschaft $\mathrm{Zu}$ " rich, No. 96, P.1-23. The English translation of this paper is available at http://sepwww.stanford.edu/sep/berryman/ PS/gassmann.pdf.

[4] Duffy, J. and Mindlin, R.D., 1957. Stress-strain relations and vibrations of a granular medium: Journal of Applied Mechanics, V. 24, p. 585-593.

[5] Varela, O.J., et al., 2006, Using time-lapse seismic amplitude data to detect variations of pore pressure and fluid saturation due to oil displacement by water: a numerical study based on one-dimensional prestack inversion: Journal of Geophysics and Engineering, p. 177. 
[6] Wood, A.W., 1955, A Textbook of Sound. New York: McMillan Co.

[7] Smith, T.M., et al., 2003, Gassmann fluid substitutions-A tutorial: Geophysics, V. 68, No. 2, p. 430-440.

[8] Geertsma, J. and Smit, D.C., 1961, Some aspects of elastic wave propagation in fluid-saturated porous solids: Geophysics, V. 26, No. 2, p. 169-181.

[9] Mindlin, R.D., 1949, Compliance of elastic bodies in contact, Journal of Applied Mechanics, No. 16, p. 259268.

[10] Andersen, C.F. and Johansen, T.A., 2010, Test of rock physics models for prediction of seismic velocities in shallow unconsolidated sands: a well log data case: Geophysical Prospecting, V. 58, No. 6, p. 1083-1098.

[11] Vidal S, 2000, Integrating geomechanics and geophysics for reservoir seismic monitoring feasibility studies, SPE 65157, SPE Annual Technical Conference and Exhibition, Paris, France, October 24-25.

[12] Landrø M, 2001, Discrimination between pressure and fluid saturation changes from time-lapse seismic data, Geophysics, 66836-844.

[13] Murphy, W.F., 1982, Effects of microstructure and pore fluids on the acoustic properties of granular sedimentary materials, Stanford University.
[14] Christensen N I and Wang H F, 1985, The Influence of pore pressure and confining pressure on dynamic elastic properties of Berea sandstone, Geophysics, 50207-213.

[15] Osdal, B., O. Husby, H. A. Aronsen, N. Chen, and T. Alsos, 2006, Mapping the fluid front and pressure buildup using 4D data on Norne Field: The Leading Edge, 25, 1134-1141, doi:10.1190/1.2349818

[16] Horne, S. and MacBeth, C., 1994, Inversion for seismic anisotropy using genetic algorithms.Geophysical Prospecting 42(8), 953 \pm 974

[17] Dal Moro, G., Pipan, M, 2007, Joint Inversion of Surface Wave Dispersion Curves and Reflection Travel Times via Multiobjective Evolutionary Algorithms, Journal of Applied Geophysics, 61, 56-81.

[18] Boomer, K., Brazier, R , 2009, Stochastic Modeling of the Velocity Structure: Beyond Joint Inversion Methods, 11th SAGA Biannual Technical Meeting and Exhibition, Switzerland, 16-18 September.

[19] Rwechungura, R., Suwartadi, E., Dadashpour, M. Kleppe J., and B. Foss, 2010, The Norne Field Case - A Unique Comparative Case Study, SPE 127538, the SPE intelligent energy conference and exhibition, Utrecht, The Netherlands, March 23-25.

[20] Dadashpour, M., Echeverría Ciaurri, D., Mukerji, T., Kleppe, J., Landrø, M., A Derivative-Free Approach for the Estimation of Porosity and Permeability Using TimeLapse Seismic and Production Data, SPE 2009. 